\title{
Flame Retardant and Mechanical Properties of Expanded Polystyrene Foams Containing Aluminum Phosphinate and Expandable Graphite
}

\author{
Lifei $\mathrm{Hu}^{\mathrm{a}}$ and Zhengzhou Wang ${ }^{\mathrm{b}}$ \\ School of Materials Science and Engineering, Tongji University, Shanghai, China, 200092 \\ a1333591@tongji.edu.cn, bzwang@tongji.edu.cn
}

\begin{abstract}
Keywords: Expanded polystyrene foam, Expandable graphite, Aluminum phosphinate Abstract. Halogen-free flame retarded expanded polystyrene (EPS) foams were prepared in this work. The effect of two kinds of aluminum phosphinates, i.e. diethyl aluminum phosphinate (DEAP) and diisobutyl aluminum phosphinate (DIAP), and their combination with expandable graphite (EG) on mechanical, flame retardant and thermal properties of EPS foams was studied. The results show that the use of DEAP or DIAP along with EG can greatly improve the flexural strength compared with same content of EG, whereas the LOI value slightly decreases. Moreover, thermal stability of EPS and flame retardant EPS foams was investigated.
\end{abstract}

\section{Introduction:}

Expanded polystyrene (EPS) foams are widely used in construction, packaging and other industries for their low cost, good thermal insulation and excellent mechanical properties ${ }^{[1]}$. However, fires are usually caused by the ignition of EPS foams leading to many deaths of people and heavy loss of properties. Nowadays, EPS foams are generally flame retarded by using halogen-containing compounds which will produce a lot of smoke and toxic gases in fires, therefore halogen-free flame retardation of EPS foams is urgently needed.

During the past decades, many different halogen-free flame retardants have been studied in EPS foams $^{[2-4]}$. Among them, expandable graphite (EG) is one of the most effective compounds for EPS foams. It is well known that the shortcoming of EG is of large particle size, which usually deteriorates mechanical properties of the foams. Aluminum phosphinates are an effective flame retardant for many polymers ${ }^{[5-6]}$.

In this work, we studied the effect of two kinds of aluminum phosphinates, i.e. diethyl aluminum phosphinate (DEAP) and diisobutyl aluminum phosphinate (DIAP), and their combination with EG on mechanical, flame retardant and thermal properties of EPS foams.

\section{Experimental}

\section{Materials}

Phosphoric acid, p-toluenesulfonic acid, n-pentane and glycerol were all purchased from Sinopharm Chemical Reagent Co. (Shanghai, China). Resol-type phenolic resin was supplied by Shandong Shengquan Chemical Co., Ltd. (Shandong, China). Expandable graphite $(150 \mu \mathrm{m})$ was provided from Tianhe graphite co., LTD (Shandong, China). Diethyl aluminum phosphinate (DEAP) and diisobutyl aluminum phosphinate (DIAP) were supplied by Jacques Jiangsu Science and Technology co., LTD (Wuxi, China).

\section{Preparation of Flame Retardant EPS foam}

$25 \mathrm{~g}$ pre-foamed EPS beads (pre-forming process: $100^{\circ} \mathrm{C}$ vapour for $2 \mathrm{~min}$; aging time: $24 \mathrm{~h}$ ) were mixed at high speeds with a prepared flame retardant adhesive (a mixture of $25 \mathrm{~g}$ phenolic resin, $2.5 \mathrm{~g}$ curing agent and a certain amount of flame retardants) until the halogen-free flame retardant adhesive evenly coated on the surface of EPS beads. Then the treated EPS beads were put into the mould and moulded at $100{ }^{\circ} \mathrm{C}$ steam environment for 25 min. After the mould cooled to room temperature, the halogen free flame retarded EPS foams were prepared. The detailed formulations of these foams were summarized in Table 1. 
Table 1 Formulations of EPS and flame retarded EPS foams

\begin{tabular}{ccccccc}
\hline Sample code & $\begin{array}{c}\text { EPS } \\
\text { [phr] }\end{array}$ & $\begin{array}{c}\text { PF resin } \\
\text { [phr] }\end{array}$ & $\begin{array}{c}\text { CA }^{a} \\
{[\mathrm{phr}]}\end{array}$ & $\begin{array}{c}\text { EG } \\
{[\mathrm{phr}]}\end{array}$ & $\begin{array}{c}\text { DEAP } \\
\text { [phr] }\end{array}$ & $\begin{array}{c}\text { DIAP } \\
\text { [phr] }\end{array}$ \\
\hline E0 & 100 & 0 & 0 & 0 & 0 & 0 \\
EPF & 100 & 125 & 12.5 & 0 & 0 & 0 \\
EEG & 100 & 125 & 12.5 & 25 & 0 & 0 \\
EGDE1 & 100 & 125 & 12.5 & 20 & 5 & 0 \\
EGDE2 & 100 & 125 & 12.5 & 15 & 10 & 0 \\
EGDE3 & 100 & 125 & 12.5 & 10 & 15 & 0 \\
EGDE4 & 100 & 125 & 12.5 & 5 & 20 & 0 \\
EDE & 100 & 125 & 12.5 & 0 & 25 & 0 \\
EGDI1 & 100 & 125 & 12.5 & 20 & 0 & 5 \\
EGDI2 & 100 & 125 & 12.5 & 15 & 0 & 10 \\
EGDI3 & 100 & 125 & 12.5 & 10 & 0 & 15 \\
EGDI3 & 100 & 125 & 12.5 & 5 & 0 & 20 \\
EDI & 100 & 125 & 12.5 & 0 & 0 & 25 \\
\hline
\end{tabular}

${ }^{a}$ Curing agent: The weight ratio of the phosphoric acid : p-toluene sulfonic acid : water $=1: 2: 2$

\section{Measurements}

Mechanical properties were tested by a universal testing machine. Flame retardant property (LOI) was carried out by oxygen index meter. Thermal gravimetric analysis (TGA) was carried out was examined by using a STD Q600 (simultaneous differential scanning calorimetry-TGA) thermo-analyzer instrument (TA Co., New Castle, DE, USA) at a heating rate of $20^{\circ} \mathrm{C} / \mathrm{min}$ under nitrogen flow.

\section{Results and discussion}

Figure 1 presents the LOI values of EPS and flame retarded EPS foams. The LOI value of pure EPS foam is $18 \%$ indicating that it is very flammable. If the EPS beads are coated by only phenolic resin, the LOI increases to $25.5 \%$. When the beads are coated by a mixture of phenolic resin and a flame retardant i.e. EG, DEAP and DIAP, respectively, the LOI value of the foams increases further. The LOI value of the foams in the presence of EG, DEAP and DIAP is $32.5 \%, 29 \%$ and $27 \%$, respectively.

If EG is combined with DEAP (or DIAP) at different ratios of EG to DEAP (or DIAP), the LOI value decreases gradually with the content of DEAP (or DIAP) in EPS foams increases, which is shown in Figure 1(a) and Figure 1(b). Above results indicate that DEAP and DIAP is not as efficient as EG in the EPS foam, and there seems no flame retardant synergistic effect between EG and DEAP (or DIAP) in the foam.
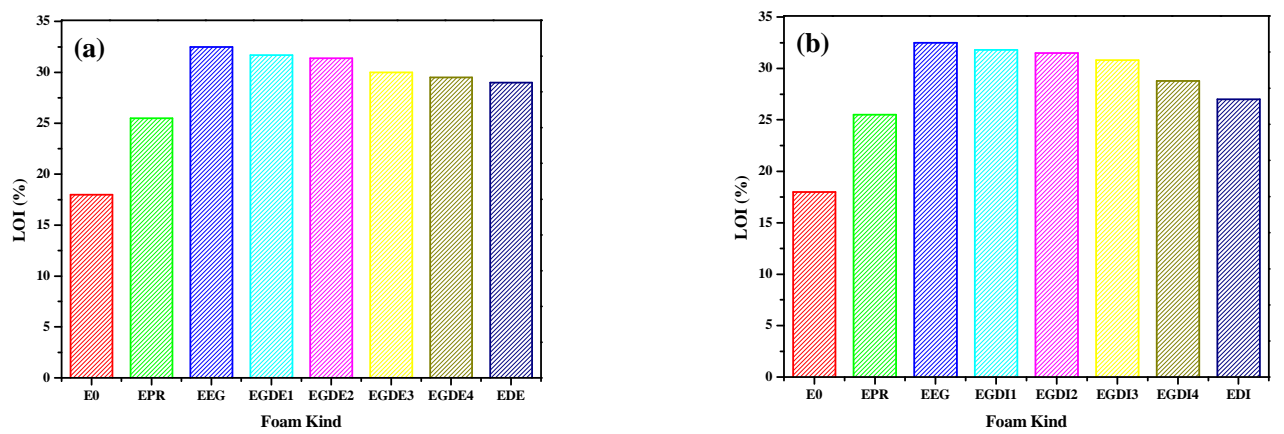

Fig. 1 LOI value of EPS and flame retardant EPS foams

Flexural strength of EPS and flame retarded EPS foams is shown in Figure 2. The coating of 
phenolic resin on the surface of EPS beads results in an about 18\% increase in the flexural strength (from $0.4 \mathrm{MPa}$ to $0.47 \mathrm{MPa}$ ) compared with pure EPS foam. If EPS beads are coated by a mixture of phenolic resin and EG, the flexural strength of the foam EEG decreases to $0.28 \mathrm{MPa}$ (ca. 30\% reduction). If EPS beads are coated by a mixture of phenolic resin and DEAP (or DIAP), the flexural strengths of the foams EDE and EDI are as high as $0.92 \mathrm{MPa}$ and $0.79 \mathrm{MPa}$, respectively. When EG is combined with DEAP (or DIAP) at different ratios of EG to DEAP (or DIAP), the flexural strengths of the foams (EGDE and EGDI series) increase gradually with the increase of the content of DEAP (or DIAP) in EPS foams, as shown in Figure 2(a) and Figure 2(b).
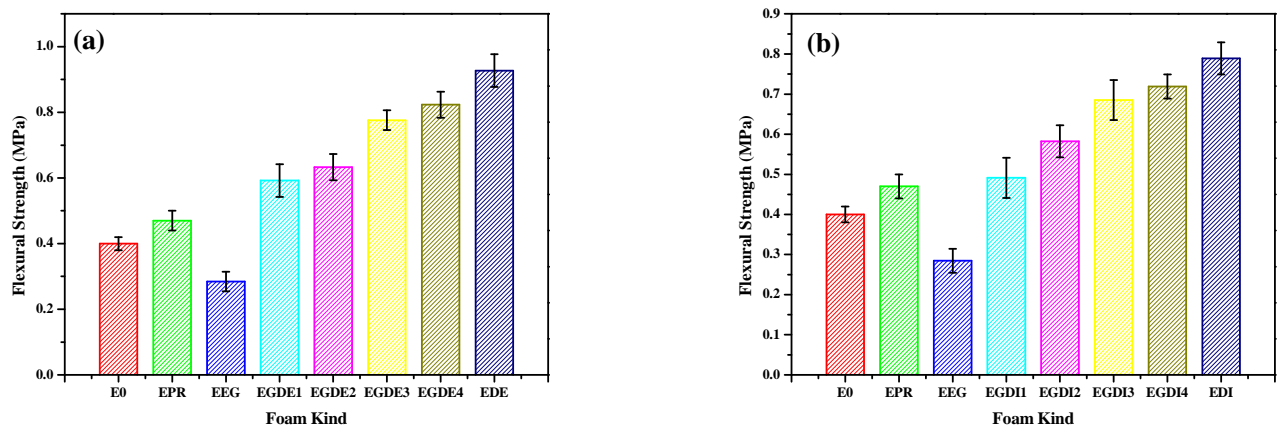

Fig. 2 Flexural strength of EPS and flame retardant EPS foams

Thermal gravimetric analysis (TGA) results are shown in Figure 3. We can see from Figure 3 that pure EPS begins to decompose at about $100^{\circ} \mathrm{C}$ and experiences a fast decomposition process from $300^{\circ} \mathrm{C}$ to $400^{\circ} \mathrm{C}$. There is almost no residue at $800^{\circ} \mathrm{C}$ for pure EPS foam. Compared with pure EPS foam, the foam treated by phenolic resin (the foam EPR) decomposes earlier, and left around 23\% residue at $800^{\circ} \mathrm{C}$. The initial thermal decomposition of the foam treated by phenolic resin and EG (the foam EEG) is quite similar. The weight loss of the foam EEG at temperature range $200^{\circ} \mathrm{C}$ to $400^{\circ} \mathrm{C}$ is much less than that of the foam EPR, and the residue at $800^{\circ} \mathrm{C}$ of the foam EEG is also higher than the one of the foam EPR. The weight loss of the foam treated by a mixture of phenolic resin, EG and aluminum phosphinate (the foams EGDE3 and EGDI3) is lower than that of the foam EEG at whole decomposition temperature range, indicating a better thermal stability of the foams EGDE3 and EGDI3 than the foam EEG.

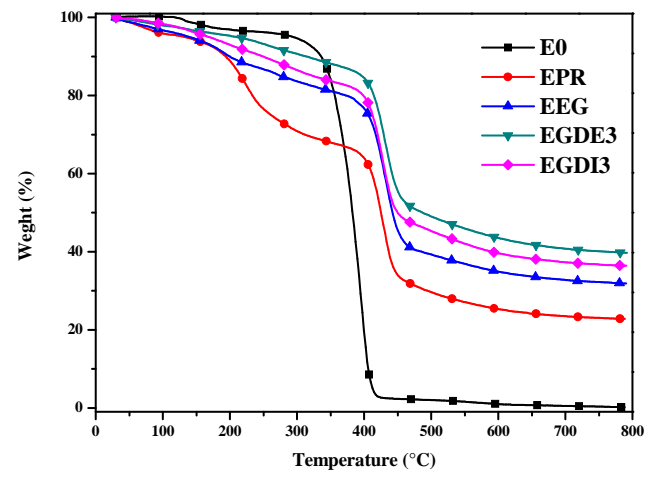

Fig. 3 TGA of EPS and flame retardant EPS foams.

\section{Conclusion}

Two kinds of aluminum phosphinates, DEAP and DIAP, combined with EG were introduced into EPS foams, and the effect of ratios of aluminum phosphinates to EG on foam properties was investigated. The LOI of the flame retarded EPS foams decreases slightly when the EG is partly substituted by DEAP or DIAP, whereas the flexural strength increases greatly. TGA results prove that the thermal stability of the foams EGDE3 and EGDI3 is higher than the one of the foam EEG. 


\section{Acknowledgements}

This work was financially supported by the National Natural Science Foundation of China (Nos. U1205114/L11 and 21174106).

\section{References}

[1] X. Chen, Y. Liu, S. Bai and Q. Wang: Polymer-Plastics Technology and Engineering Vol. 53 (2014), p. 1402-1407.

[2] N. Cinausero, N. Azema, J. M. Lopez-Cuesta, M. Cochez and M. Ferriol: Polymer Degradation and Stability Vol. 96 (2011), p. 1445-1454.

[3] D. Price, L. K. Cunliffe, K. J. Bullett, T. R. Hull, G. J. Milnes, J. R. Ebdon, B. J. Hunt and P. Joseph: Polymer Degradation and Stability Vol. 92 (2007), p. 1101-1114.

[4] Y.-T.-H. Quach, L. Ferry, R. Sonnier and J.-M. Lopez Cuesta: Polymers for Advanced Technologies Vol. 24 (2013), p. 104-113.

[5] N. Wu and X. Li: Polymer Degradation and Stability Vol. 105 (2014), p. 265-276.

[6] H. Yang, X. Wang, L. Song, B. Yu, Y. Yuan, Y. Hu and R. K. K. Yuen: Polymers for Advanced Technologies Vol. 25 (2014), p. 1034-1043. 